\title{
EGU21-2712
}

EGU General Assembly 2021

(c) Author(s) 2021. This work is distributed under

the Creative Commons Attribution 4.0 License.

\section{Innovative methods for earthquake damage detection and classification using airborne observation of critical infrastructures (project LOKI)}

\author{
Julia Kohns ${ }^{1}$, Vivien Zahs ${ }^{2}$, Tahira Ullah ${ }^{3}$, Danijel Schorlemmer ${ }^{4}$, Cecilia Nievas $^{4}$, Katharina Glock ${ }^{5}$, \\ Fabian Meyer ${ }^{5}$, Heiko Mey ${ }^{6}$, Lothar Stempniewski ${ }^{1}$, Benjamin Herfort ${ }^{3,8}$, Alexander Zipf ${ }^{3,8,7}$, and \\ Bernhard Höfle ${ }^{2,7}$ \\ ${ }^{1}$ Institute of Reinforced Concrete Structures and Building Materials, Karlsruhe Institute of Technology, Karlsruhe, Germany \\ (julia.kohns@kit.edu) \\ 23DGeo Research Group, Heidelberg University, Heidelberg, Germany \\ ${ }^{3}$ GIScience Research Group, Heidelberg University, Heidelberg, Germany \\ ${ }^{4}$ GFZ German Research Centre for Geosciences, Potsdam, Germany \\ ${ }^{5} \mathrm{FZI}$ Research Center for Information Technology, Karlsruhe, Germany \\ ${ }^{6}$ Aeromey $\mathrm{GmbH}$, Bensheim, Germany \\ ${ }^{7}$ Interdisciplinary Center for Scientific Computing, Heidelberg University, Heidelberg, Germany \\ ${ }^{8}$ Heidelberg Institute for Geoinformation Technology gGmbH, Heidelberg University, Heidelberg, Germany
}

Earthquakes play a major role worldwide regarding economic and social consequences. In the event of an earthquake, many lives are at risk and the impact on the built and natural environment may be significant. Until now, estimations of damage and losses and the assessment of the stability of buildings are, however, only available several days to months after the event and are often based on the subjective assessment of experienced engineers.

For the effective planning of rescue measures and the best possible use of available resources, a fast, (semi-)automatic and accurate detection of the situation and an objective assessment of damage to critical infrastructures is indispensable. This requires a combination of innovative methods and technologies (UAVs, Machine Learning and Crowdsourcing combined with earthquake engineering knowledge) covering a wide range of spatial and temporal scales.

The interdisciplinary system LOKI (www.uni-heidelberg.de/loki) consists of the following procedure: After the occurrence of an earthquake, an initial damage forecast is made within a few minutes based on the Global Dynamic Exposure model and integrated vulnerability functions in combination with the ground-motion field to identify areas with potential high/low damage. Missing building footprints and required building information are recorded via a crowdsourcing approach to complete the OpenStreetMap building database, which serves as input to the exposure model. In parallel, mission plans for overview flights are created and transferred to fixedwing UAVs, which record low to medium-resolution photos and 3D point clouds of the entire affected area. These data are used for damage detection, in which a binary distinction is made at building level between visible and non-visible damage using Machine Learning approaches. Thus, 
after a few hours, first orthophotos and the location of potentially damaged buildings can already be transmitted to emergency response teams. Thereafter, mission planning focuses on the capture of high-resolution 3D information of individual buildings. Fleets of multicopter drones provide highly detailed 3D imagery following mission plans that can be modified in real time by the emergency response teams. The mission planning algorithms support prioritization of specific areas or buildings for data acquisition, so that rescue measures can be optimally supported. The acquired high-resolution images and point clouds serve as input for damage classification, which is carried out per building using a combination of automatic procedures and Micro-Mapping. This offers the possibility to combine the advantages of fast automated procedures with the human ability to visually interpret details. Potential global and building material-related damage characteristics, which are based on observations of previous earthquakes, are included in a damage catalogue and allow building damage to be classified into five damage grades. In an iterative process, a timely and objective building-level classification of damage with an indication of the reliability of the specified degree of damage is achieved.

The integration of various disciplines and the combination of different concepts and technologies allows supporting disaster relief in different temporal and spatial resolutions with timely and reliable information on earthquake-induced damage. 